\title{
On the Rate of Convergence by Generalized Baskakov Operators
}

\author{
Yi Gao, ${ }^{1}$ Wenshuai Wang, ${ }^{2}$ and Shigang $Y^{3} e^{3}$ \\ ${ }^{1}$ School of Mathematics and Information Science, Beifang University of Nationalities, Yinchuan, Ningxia 750021, China \\ ${ }^{2}$ School of Mathematics and Computer Science, Ningxia University, Yinchuan, Ningxia 750021, China \\ ${ }^{3}$ School of Computer Science, University of Lincoln, Lincoln LN6 7TS, UK
}

Correspondence should be addressed to Wenshuai Wang;wws@nxu.edu.cn

Received 19 December 2014; Accepted 10 March 2015

Academic Editor: Hagen Neidhardt

Copyright (C) 2015 Yi Gao et al. This is an open access article distributed under the Creative Commons Attribution License, which permits unrestricted use, distribution, and reproduction in any medium, provided the original work is properly cited.

We firstly construct generalized Baskakov operators $V_{n, \alpha, q}(f ; x)$ and their truncated sum $B_{n, \alpha, q}\left(f ; \gamma_{n}, x\right)$. Secondly, we study the pointwise convergence and the uniform convergence of the operators $V_{n, \alpha, q}(f ; x)$, respectively, and estimate that the rate of convergence by the operators $V_{n, \alpha, q}(f ; x)$ is $1 / n^{q / 2}$. Finally, we study the convergence by the truncated operators $B_{n, \alpha, q}\left(f ; \gamma_{n}, x\right)$ and state that the finite truncated sum $B_{n, \alpha, q}\left(f ; \gamma_{n}, x\right)$ can replace the operators $V_{n, \alpha, q}(f ; x)$ in the computational point of view provided that $\lim _{n \rightarrow \infty} \sqrt{n} \gamma_{n}=\infty$.

\section{Introduction}

Let $N=\{1,2, \ldots\}, N_{0}=N \cup\{0\}, R_{+}=(0,+\infty)$, and $R_{0}=R_{+} \cup$ $\{0\}$. For a fixed $q \in N_{0}$, we introduce the weighted function $w_{q}$ on $R_{0}$ by

$$
w_{q}(x)= \begin{cases}1, & q=0, \\ \left(1+x^{q}\right)^{-1}, & q \in N .\end{cases}
$$

Associated with the above weighted function, we also introduce the polynomial weighted space $S_{q}$ of all real-valued continuous functions $f$ on $R_{0}$ for which $w_{q} f$ is uniformly continuous and bounded on $R_{0}$, and the norm on $S_{q}$ is defined by the formula

$$
\|f\|_{q, \infty}=\sup _{x \in R_{0}} w_{q}(x)|f(x)| .
$$

Obviously, when $q=0$, then the above norm is the ordinary norm $\|f\|_{\infty}$. Furthermore, for fixed $q \in N_{0}$, let $S_{q}^{q}$ be the set of all functions $f \in S_{q}$ for which $w_{q-k}(x) f^{(k)}(x)(k=$ $0,1,2, \ldots, q)$ are continuous and bounded on $R_{0}$ and $f^{(q)}$ is uniformly continuous on $R_{0}$, where $f^{(k)}(x)(k=0,1,2, \ldots, q)$ denote the $k$ th order derivative of $f$ on $R_{0}$.
Let $f$ be a function defined on $R_{0}$; Baskakov [1] introduced the sequence of linear positive operators $V_{n}(f ; x)$ as follows:

$$
V_{n}(f ; x)=\sum_{k=0}^{\infty} b_{n, k}(x) f\left(\frac{k}{n}\right),
$$

where $b_{n, k}(x)$ is called a Baskakov operator's kernel, which is defined by

$$
b_{n, k}(x)=\frac{n(n+1) \cdots(n+k-1)}{k !} x^{k}(1+x)^{-n-k} .
$$

Based on the Baskakov operators, many Baskakov-type operators [2-13] and their multivariate Baskakov operators [11, 14-18] were discussed. Particularly, Gupta and Agarwal studied the Baskakov-Kantorovich operators, SzászBaskakov operators, and so forth in their recent book [6]. One of the most famous Baskakov-type operators is called generalized Baskakov operators [19-22]. One has

$$
V_{n, \alpha}(f ; x)=\sum_{k=0}^{\infty} b_{n, k, \alpha}(x) f\left(\frac{k}{n}\right)
$$


where

$$
\begin{aligned}
b_{n, k, \alpha}(x)= & \frac{n(n+\alpha) \cdots(n+(k-1) \alpha)}{k !} \\
& \cdot x^{k}(1+\alpha x)^{-n / \alpha-k}, \quad \alpha>0 .
\end{aligned}
$$

Other modified Baskakov operators are defined as follows [10]:

$$
V_{n, q}(f ; x)=\sum_{k=0}^{\infty} b_{n, k}(x) \sum_{i=0}^{q} \frac{f^{(i)}(k / n)}{i !}\left(x-\frac{k}{n}\right)^{i},
$$

$x \in R_{0}, q \in N$.

By combining the above operators (5) with (7), we introduce the following class of operators.

Definition 1. For $x \in R_{0}$ and $q \in N$, other generalized Baskakov-type operators are defined by

$$
V_{n, \alpha, q}(f ; x)=\sum_{k=0}^{\infty} b_{n, k, \alpha}(x) \sum_{i=0}^{q} \frac{f^{(i)}(k / n)}{i !}\left(x-\frac{k}{n}\right)^{i} .
$$

The actual construction of Baskakov operator and its various modifications requires estimations of infinite series which in a certain sense restrict their usefulness from the computational point of view. A question naturally arises of whether the Baskakov operators can be replaced by a finite sum. In connection with this question we construct a new family of linear positive operators as follows:

$$
B_{n, \alpha, q}\left(f ; \gamma_{n}, x\right)=\sum_{k=0}^{\left[n\left(x+\gamma_{n}\right)\right]} b_{n, k, \alpha}(x) \sum_{i=0}^{q} \frac{f^{(i)}(k / n)}{i !}\left(x-\frac{k}{n}\right)^{i},
$$

where $\left\{\gamma_{n}\right\}_{n=1}^{\infty}$ is a sequence of positive numbers such that $\lim _{n \rightarrow \infty} \sqrt{n} \gamma_{n}=\infty$ and $\left[n\left(x+\gamma_{n}\right)\right]$ denotes the integral part of $n\left(x+\gamma_{n}\right)$.

Obviously, when $\alpha=1$ and $q=0$, the operators (8) are (5), while the operators (9) are degenerated as follows, which are firstly proposed by Walczak [11]:

$$
A_{n}\left(f ; \gamma_{n}, x\right)=\sum_{k=0}^{\left[n\left(x+\gamma_{n}\right)\right]} b_{n, k}(x) f\left(\frac{k}{n}\right)
$$

And when $\alpha=1$, the operators (8) are (7), while the operators (9) can be represented by [12]

$$
F_{n, q}\left(f ; \gamma_{n}, x\right)=\sum_{k=0}^{\left[n\left(x+\gamma_{n}\right)\right]} b_{n, k}(x) \sum_{i=0}^{q} \frac{f^{(i)}(k / n)}{i !}\left(x-\frac{k}{n}\right)^{i} .
$$

For the convenience of discussion in the rest of paper, we use the notation that $K_{n, \alpha, q}\left(f ; \gamma_{n}, x\right)$ denotes the remainder term of operators $V_{n, \alpha, q}(f ; x)$ associated with the truncated $\operatorname{sum} B_{n, \alpha, q}\left(f ; \gamma_{n}, x\right)$. Consider

$$
\begin{aligned}
K_{n, \alpha, q} & \left(f ; \gamma_{n}, x\right) \\
& =\sum_{k=\left[n\left(x+\gamma_{n}\right)\right]+1}^{\infty} b_{n, k, \alpha}(x) \sum_{i=0}^{q} \frac{f^{(i)}(k / n)}{i !}\left(x-\frac{k}{n}\right)^{i} .
\end{aligned}
$$

This paper focuses on convergence of the operators $V_{n, \alpha, q}(f ; x)$ and their truncated sum $B_{n, \alpha, q}\left(f ; \gamma_{n}, x\right)$. The rest of the paper is organized as follows. In Section 2, we give main lemmas and prove that the remainder term $K_{n, \alpha, q}\left(f ; \gamma_{n}, x\right)$ of the operators $V_{n, \alpha, q}(f ; x)$ associated with the truncated sum $B_{n, \alpha, q}\left(f ; \gamma_{n}, x\right)$ is convergent to 0 provided that $\lim _{n \rightarrow \infty} \sqrt{n} \gamma_{n}=\infty$. In Section 3, we state the pointwise convergence and the uniform convergence of the operators $V_{n, \alpha, q}(f ; x)$ on the polynomial weighted space $S_{q}$, respectively, which indicate that the rate of convergence by the operators $V_{n, \alpha, q}(f ; x)$ is $1 / n^{q / 2}$. Finally, we study the convergence by the truncated operators $B_{n, \alpha, q}\left(f ; \gamma_{n}, x\right)$ and state that the finite truncated sum $B_{n, \alpha, q}\left(f ; \gamma_{n}, x\right)$ can replace the operators $V_{n, \alpha, q}(f ; x)$ in the computational point of view.

In this paper, for better characterizing the degree of approximation by the generalized Baskakov operators $V_{n, \alpha, q}(f ; x)$, we introduce the classical modulus of continuity of a function $f \in S_{q}$, defined by [23]

$$
w(f ; t):=\sup _{0 \leq h \leq t}\|f(\cdot+h)-f(\cdot)\|_{\infty}, \quad t \in R_{0} .
$$

Here, we give an important property of modulus of continuity, which will be used in the proof of Theorem 6 . One has

$$
w(f ; t) \leq\left(1+\frac{t}{\delta}\right) w(f ; \delta), \quad \delta \in R_{0}
$$

\section{Main Lemmas}

In this section, we give some properties of the above operators, which will be used to prove the main theorems.

Lemma 2 (see [22]). If $V_{n, \alpha}(f ; x)$ is defined by formula (5) then

$$
\begin{gathered}
V_{n, \alpha}(1 ; x)=1 ; \quad V_{n, \alpha}(t ; x)=x ; \\
V_{n, \alpha}\left((t-x)^{2} ; x\right)=\frac{x(1+\alpha x)}{n} .
\end{gathered}
$$

From the first equality in Lemma 2, for all $f(x), x \in R_{0}$, we have $f(x)=V_{n, \alpha}(f(x) ; x)$.

Lemma 3 (see [19]). If $V_{n, \alpha}(f ; x)$ is defined by formula (5), for fixed $2 \leq q \in N$, there exist $m \leq q$-order algebraic polynomials $P_{i, q, \alpha}, 0 \leq i \leq q$, with coefficients depending only on $i, q, \alpha$, such that

$$
V_{n, \alpha}\left((t-x)^{q} ; x\right)=\sum_{i=0}^{[q / 2]} \frac{P_{i, q, \alpha}(x)}{n^{q-i}}
$$

where $x \in R_{0}$ and $[q / 2]$ denotes the integral part of $q / 2$. Moreover,

$$
V_{n, \alpha}\left((t-x)^{2 m} ; x\right) \leq C\left(\frac{x(1+\alpha x)}{n}+\frac{1}{n^{2}}\right)^{m}, \quad m \in N
$$

Here and in the rest of the paper, $C$ denotes a positive absolute constant, whose value may change from line to line but is independent of $n$. 
For example, when $q=4$, we have the following 4-order algebraic polynomial:

$$
\begin{aligned}
& V_{n, \alpha}\left((t-x)^{4} ; x\right) \\
& =3\left[\left(\frac{\alpha}{n}\right)^{2}+2\left(\frac{\alpha}{n}\right)^{2}\right] x^{4}+\frac{6}{n}\left[\frac{\alpha}{n}+2\left(\frac{\alpha}{n}\right)^{2}\right] x^{3} \\
& \quad+\frac{1}{n^{2}}\left(3+\frac{\alpha}{n}\right) x^{2}+\frac{1}{n^{3}} x .
\end{aligned}
$$

For fixed $x_{0} \in R_{+}$, obviously, we have

$$
V_{n, \alpha}\left((t-x)^{4} ; x\right)=O_{x_{0}}\left(\frac{1}{n^{2}}\right)
$$

Furthermore, with respect to the above weighted function $w_{q}(x)$, the generalized Baskakov operators (5) have the following results, which demonstrate that the weighted function $w_{q}(x)$ is also important to the generalized Baskakov operators.

Lemma 4 (see $[15,21])$. If $V_{n, \alpha}(f ; x)$ and weighted function $w_{q}(x)$ are defined by formula (5) and (1), respectively, for $x \in$ $R_{+}$, then there exist positive absolute constants $C$, such that

$$
\begin{gathered}
w_{q}(x) V_{n, \alpha}\left(\frac{1}{w_{q}(t)} ; x\right) \leq C \\
w_{q}(x) V_{n, \alpha}\left(\frac{(t-x)^{2}}{w_{q}(t)} ; x\right) \leq C \frac{x(1+\alpha x)}{n} .
\end{gathered}
$$

Now we will give the estimation of $K_{n, \alpha, q}\left(f ; \gamma_{n}, x\right)$.

Lemma 5. For $f \in S_{q}^{q}, q \in N, K_{n, \alpha, q}\left(f ; \gamma_{n}, x\right)$ is defied by (12), then

$$
\begin{aligned}
& \left|K_{n, \alpha, q}\left(f ; \gamma_{n}, x\right)\right| \\
& \leq 2^{q} C\left(\frac{x(1+\alpha x)}{n}+\frac{1}{n^{2}}\right)^{q / 2} \\
& \quad+C \sum_{i=0}^{q} \frac{1+2^{q-i-1} x^{q-i}}{\gamma_{n}^{q}}\left(\frac{x(1+\alpha x)}{n}+\frac{1}{n^{2}}\right)^{(q+i) / 2} .
\end{aligned}
$$

Furthermore, one has

$$
\lim _{n \rightarrow \infty} K_{n, \alpha, q}\left(f ; \gamma_{n}, x\right)=0 .
$$

Proof. By assumption $f \in S_{q}^{q}$, there is a positive absolute constant $C$, such that $\left|f^{(i)}(t)\right| \leq C\left(1+t^{q-i}\right), i=0,1, \ldots, q$. With the elementary inequality $(a+b)^{k} \leq 2^{k-1}\left(a^{k}+b^{k}\right)$ for $a, b \in R_{+}, k \in N$, we get

$$
\begin{aligned}
\left|f^{(i)}(t)\right| & \leq C\left(1+(|t-x|+x)^{q-i}\right) \\
& \leq C\left(1+2^{q-i-1}\left(|t-x|^{q-i}+x^{q-i}\right)\right) .
\end{aligned}
$$

So we have

$$
\begin{aligned}
\left|K_{n, \alpha, q}\left(f ; \gamma_{n}, x\right)\right| & \\
\leq & C \sum_{k=\left[n\left(x+\gamma_{n}\right)\right]+1}^{\infty} b_{n, k, \alpha}(x) \\
& \cdot \sum_{i=0}^{q} \frac{1}{i !}\left(1+2^{q-i-1}\left(\left|\frac{k}{n}-x\right|^{q-i}+x^{q-i}\right)\right)\left|\frac{k}{n}-x\right|^{i} \\
\leq & C \sum_{i=0}^{q} 2^{q-i-1} \sum_{k=\left[n\left(x+\gamma_{n}\right)\right]+1}^{\infty} b_{n, k, \alpha}(x)\left|\frac{k}{n}-x\right|^{q} \\
& +C \sum_{i=0}^{q}\left(1+2^{q-i-1} x^{q-i}\right) \\
\leq & C 2^{q} V_{n, \alpha}\left(|t-x|^{q} ; x\right)+C \sum_{i=0}^{\infty}\left(1+2^{q-i-1} x^{q-i}\right) \\
& \cdot \sum_{k=\left[n\left(x+\gamma_{n}\right)\right]+1}^{\infty} b_{n, k, \alpha}(x)\left|\frac{k}{n}-x\right|^{i} .
\end{aligned}
$$

Next, we estimate the sum of the last term, since $k>n\left(x+\gamma_{n}\right)$ in the last term; for $i=0,1, \ldots, q$, we remark that

$$
\begin{aligned}
& \sum_{k=\left[n\left(x+\gamma_{n}\right)\right]+1}^{\infty} b_{n, k, \alpha}(x)\left|\frac{k}{n}-x\right|^{i} \\
& \quad \leq \sum_{\gamma_{n}<|k / n-x|}^{\infty} b_{n, k, \alpha}(x)\left|\frac{k}{n}-x\right|^{i} \\
& \leq \frac{1}{\gamma_{n}^{q}} \sum_{\gamma_{n}<|k / n-x|}^{\infty} b_{n, k, \alpha}(x)\left|\frac{k}{n}-x\right|^{q+i} \\
& \leq \frac{1}{\gamma_{n}^{q}} V_{n, \alpha}\left(|t-x|^{q+i} ; x\right) .
\end{aligned}
$$

Finally, using Hölder inequality with Lemmas 2 and 3, we get the following inequality:

$$
\begin{aligned}
\left|K_{n, \alpha, q}\left(f ; \gamma_{n}, x\right)\right| & \leq 2^{q} C\left(V_{n, \alpha}\left((t-x)^{2 q} ; x\right)\right)^{1 / 2} \\
& +C \sum_{i=0}^{q} \frac{1+2^{q-i-1} x^{q-i}}{\gamma_{n}^{q}}\left(V_{n, \alpha}\left((t-x)^{2(q+i)} ; x\right)\right)^{1 / 2} \\
\leq & 2^{q} C\left(\frac{x(1+\alpha x)}{n}+\frac{1}{n^{2}}\right)^{q / 2} \\
& +C \sum_{i=0}^{q} \frac{1+2^{q-i-1} x^{q-i}}{\gamma_{n}^{q}}\left(\frac{x(1+\alpha x)}{n}+\frac{1}{n^{2}}\right)^{(q+i) / 2} .
\end{aligned}
$$


Fixing $x \in R_{0}$, there exist constants $C(x)$ that maybe depend on $x$ and constants $\alpha, q$ but are independent of $n$, such that

$$
\begin{aligned}
\mid K_{n, \alpha, q} & \left(f ; \gamma_{n}, x\right) \mid \\
\leq & 2^{q} C\left(\frac{x(1+\alpha x)}{n}+\frac{1}{n^{2}}\right)^{q / 2} \\
& +\frac{C}{\gamma_{n}^{q}} \sum_{i=0}^{q}\left(1+2^{q-i-1} x^{q-i}\right)\left(\frac{x(1+\alpha x)}{n}+\frac{1}{n^{2}}\right)^{(q+i) / 2} \\
\leq & \frac{C(x)}{n^{q / 2}}+\frac{C(x)}{n^{q / 2} \gamma_{n}^{q}},
\end{aligned}
$$

and noticing that $\lim _{n \rightarrow \infty} \sqrt{n} \gamma_{n}=\infty$, then we can get $K_{n, \alpha, q}\left(f ; \gamma_{n}, x\right)=o(1), n \rightarrow \infty$.

\section{Main Results}

In this section, we will study the properties of the operators $V_{n, \alpha, q}(f ; x)$ and give the estimation of degree of approximation by these operators.

Theorem 6. Fix $q \in N_{0}$, for every $f \in S_{2 q+1}^{2 q+1}$; then there exists a positive absolute constant $C$, such that

$$
\begin{aligned}
w_{2 q+1} & (x)\left|V_{n, \alpha, 2 q+1}(f ; x)-f(x)\right| \\
& \leq \frac{C}{(2 q+1) !}\left[\frac{1}{n^{q+1 / 2}}+\frac{1+\alpha x}{n^{q}}\right] \omega\left(f^{(2 q+1)} ; \frac{1}{n}\right),
\end{aligned}
$$

where $C>0$ is dependent only on $q$ and $\alpha$ but is independent of $x$ and $n$.

Proof. By assumption, using the modified Taylor formula [10],

$$
\begin{aligned}
f(x)= & \sum_{i=0}^{2 q+1} \frac{f^{i}(k / n)}{i !}\left(x-\frac{k}{n}\right)^{i}+\frac{(x-k / n)^{2 q+1}}{(2 q) !} \\
& \cdot \int_{0}^{1}(1-t)^{2 q} \\
& \cdot\left(f^{(2 q+1)}\left(\frac{k}{n}+t\left(x-\frac{k}{n}\right)\right)-f^{(2 q+1)}\left(\frac{k}{n}\right)\right) d t,
\end{aligned}
$$

with Lemma 2 and inequality (14), we get

$$
\begin{array}{r}
\left|V_{n, \alpha, 2 q+1}(f ; x)-f(x)\right| \\
=\mid \sum_{k=0}^{\infty} b_{n, k, \alpha}(x) \frac{(x-k / n)^{2 q+1}}{(2 q) !} \\
\quad \cdot \int_{0}^{1}(1-t)^{2 q}\left(f^{(2 q+1)}\left(\frac{k}{n}+t\left(x-\frac{k}{n}\right)\right)\right. \\
\left.-f^{(2 q+1)}\left(\frac{k}{n}\right)\right) d t \mid
\end{array}
$$

$$
\begin{aligned}
\leq & \sum_{k=0}^{\infty} b_{n, k, \alpha}(x) \frac{|x-k / n|^{2 q+1}}{(2 q) !} \\
& \cdot \int_{0}^{1}(1-t)^{2 q} \mid f^{(2 q+1)}\left(\frac{k}{n}+t\left(x-\frac{k}{n}\right)\right) \\
\leq & -f^{(2 q+1)}\left(\frac{k}{n}\right) \mid d t \\
& \sum_{k=0}^{\infty} b_{n, k, \alpha}(x) \frac{|x-k / n|^{2 q+1}}{(2 q) !} \\
& \cdot \int_{0}^{1}(1-t)^{2 q} \omega\left(f^{(2 q+1)} ;\left|t\left(x-\frac{k}{n}\right)\right|\right) d t \\
\leq & \sum_{k=0}^{\infty} b_{n, k, \alpha}(x) \frac{|x-k / n|^{2 q+1}}{(2 q) !} \\
& \cdot \int_{0}^{1}(1-t)^{2 q}\left(1+n t\left|x-\frac{k}{n}\right|\right) \omega\left(f^{(2 q+1)} ; \frac{1}{n}\right) d t \\
= & \frac{\omega\left(f^{(2 q+1)} ; 1 / n\right)}{(2 q+1) !} V_{n, \alpha}\left(|x-t|^{2 q+1} ; x\right) \\
& +n\left(f^{(2 q+1)} ; 1 / n\right) \\
& (2 q) ! \\
&
\end{aligned}
$$

where $B(a, b)(a>0, b>0)$ denotes the Beta function, $B(2,2 q+1)=1 /((2 q+1)(2 q+2))$. Using the Hölder inequality with Lemmas 2 and 3, we further have

$$
\begin{aligned}
\left|V_{n, \alpha, 2 q+1}(f ; x)-f(x)\right| & \frac{\omega\left(f^{(2 q+1)} ; 1 / n\right)}{(2 q+1) !}\left(V_{n, \alpha}\left((x-t)^{4 q+2} ; x\right)\right)^{1 / 2} \\
\leq & +n \frac{\omega\left(f^{(2 q+1)} ; 1 / n\right)}{(2 q+1) !} V_{n, \alpha}\left((x-t)^{2 q+2} ; x\right) \\
= & \frac{\omega\left(f^{(2 q+1)} ; 1 / n\right)}{(2 q+1) !} \\
& \cdot\left(\left(\sum_{j=0}^{2 q+1} \frac{P_{j, 4 q+2, \alpha}(x)}{n^{4 q+2-j}}\right)^{1 / 2}+n \sum_{j=0}^{q+1} \frac{P_{j, 2 q+2, \alpha}(x)}{n^{2 q+2-j}}\right) \\
= & \frac{\omega\left(f^{(2 q+1)} ; 1 / n\right)}{(2 q+1) !} \\
& \cdot\left(\frac{1}{n^{q+1 / 2}}\left(\sum_{j=0}^{2 q+1} P_{j, 4 q+2, \alpha}(x)\right)^{1 / 2} P_{j, 2 q+2, \alpha}(x)\right) .
\end{aligned}
$$


Thus, we obtain

$$
\begin{gathered}
w_{2 q+1}(x)\left|V_{n, \alpha, 2 q+1}(f ; x)-f(x)\right| \\
=\frac{\omega\left(f^{(2 q+1)} ; 1 / n\right)}{(2 q+1) !}\left(\frac{1}{n^{q+1 / 2}}\left(\sum_{j=0}^{2 q+1} \frac{P_{j, 4 q+2, \alpha}(x)}{\left(1+x^{2 q+1}\right)^{2}}\right)^{1 / 2}\right. \\
\left.+\frac{1}{n^{q}} \sum_{j=0}^{q+1} \frac{P_{j, 2 q+2, \alpha}(x)}{1+x^{2 q+1}}\right) .
\end{gathered}
$$

Because $P_{j, 4 q+2, \alpha}(x)$ denotes an algebraic polynomial with order at most $4 q+2$, there exists a positive absolute constant $C$, such that $\left|P_{j, 4 q+2, \alpha}(x) /\left(1+x^{2 q+1}\right)^{2}\right| \leq C$, while $P_{j, 2 q+2, \alpha}(x) /(1+$ $\left.x^{2 q+1}\right)$ is an at most 1 -order algebraic polynomial with respect to $x$; that is, there exists a positive absolute constant $C$ depending on $\alpha$ and $q$, such that $\sum_{j=0}^{q+1}\left(P_{j, 2 q+2, \alpha}(x) /(1+\right.$ $\left.\left.x^{2 q+1}\right)\right) \leq C(1+\alpha x)$.

Remark 7. The result of $V_{n, \alpha, 2 q+2}(f ; x)$ can be easily obtained by imitating Theorem 6 ; here we omit it because it will be mentioned in the proof of next theorem.

Theorem 6 is to focus on the pointwise approximation of the operators $V_{n, \alpha, q}(f ; x)$; now we will study their uniform approximation.

Theorem 8. Fix $q \in N_{0}$; for every $f \in S_{q}^{q}$, one has

$$
\left\|V_{n, \alpha, q}(f ; \cdot)-f(\cdot)\right\|_{q, \infty}=O\left(\frac{1}{q ! n^{q / 2}}\right) .
$$

Proof. From the proof of Theorem 6, for $f \in S_{2 q+1}^{2 q+1}$, we can get

$$
\begin{aligned}
& \left|V_{n, \alpha, 2 q+1}(f ; x)-f(x)\right| \\
& \leq 2\left\|f^{(2 q+1)}\right\|_{\infty} \sum_{k=0}^{\infty} b_{n, k, \alpha}(x) \frac{|x-k / n|^{2 q+1}}{(2 q+1) !} \\
& \quad=\frac{2\left\|f^{(2 q+1)}\right\|_{\infty}}{(2 q+1) !} V_{n, \alpha}\left(|x-t|^{2 q+1} ; x\right) .
\end{aligned}
$$

Using the Hölder inequality with Lemma 3, we obtain

$$
\begin{aligned}
w_{2 q+1}(x)\left|V_{n, \alpha, 2 q+1}(f ; x)-f(x)\right| \\
\leq \frac{2\left\|f^{(2 q+1)}\right\|_{\infty}}{(2 q+1) !} \frac{1}{n^{q+1 / 2}}\left(\sum_{j=0}^{2 q+1} \frac{P_{j, 4 q+2, \alpha}(x)}{\left(1+x^{2 q+1}\right)^{2}}\right)^{1 / 2} \\
\leq \frac{2 C\left\|f^{(2 q+1)}\right\|_{\infty}}{(2 q+1) !} \frac{1}{n^{q+1 / 2}} .
\end{aligned}
$$

For all $x \in R_{0}$, we have

$$
\left\|V_{n, \alpha, 2 q+1}(f ; \cdot)-f(\cdot)\right\|_{q, \infty} \leq \frac{2 C\left\|f^{(2 q+1)}\right\|_{\infty}}{(2 q+1) !} \frac{1}{n^{q+1 / 2}} .
$$

On the other hand, for $f \in S_{2 q+2}^{2 q+2}$, similar to the proof of Theorem 6, we get

$$
\left|V_{n, \alpha, 2 q+2}(f ; x)-f(x)\right| \leq \frac{2\left\|f^{(2 q+2)}\right\|_{\infty}}{(2 q+2) !} V_{n, \alpha}\left((x-t)^{2 q+2} ; x\right) .
$$

By Lemma 3, we obtain

$$
\begin{aligned}
w_{2 q+2}(x)\left|V_{n, \alpha, 2 q+2}(f ; x)-f(x)\right| \\
\leq \frac{2\left\|f^{(2 q+2)}\right\|_{\infty} \sum_{j=0}^{q+1} \frac{1}{n^{2 q+2-j}} \frac{P_{j, 2 q+2, \alpha}(x)}{1+x^{2 q+2}}}{\leq} \\
\leq \frac{2 C\left\|f^{(2 q+2)}\right\|_{\infty}}{(2 q+2) !} \frac{1}{n^{q+1}} .
\end{aligned}
$$

For all $x \in R_{0}$, we further have

$$
\left\|V_{n, \alpha, 2 q+2}(f ; \cdot)-f(\cdot)\right\|_{q, \infty} \leq \frac{2 C\left\|f^{(2 q+2)}\right\|_{\infty}}{(2 q+2) !} \frac{1}{n^{q+1}} .
$$

Combining the above two inequalities (36) and (39), for all $f \in S_{q}^{q}$ and fixed $q \in N_{0}$, the desired equality (33) is obtained.

Remark 9. Theorem 8 indicates that the rate of convergence by the operator $V_{n, \alpha, q}(f ; x)$ is $1 / n^{q / 2}$.

Corollary 10. Let $f \in S_{q}^{q}$ with some $q \in N_{0}$, for all $x \in R_{+}$; then

$$
\lim _{n \rightarrow \infty} V_{n, \alpha, q}(f ; x)=f(x) .
$$

Finally, we will discuss the convergence of the truncated $\operatorname{sum} B_{n, \alpha, q}\left(f ; \gamma_{n}, x\right)$.

Theorem 11. Let $f \in S_{q}^{q}$ with some $q \in N_{0}$, for fixed $x \in R_{+}$; then

$$
\lim _{n \rightarrow \infty} B_{n, \alpha, q}\left(f ; \gamma_{n}, x\right)=f(x)
$$

Moreover, the assertion (41) holds uniformly on every rectangle $x \in[a, b]$ with $0<a<b$.

Proof. Notice that

$$
\begin{aligned}
B_{n, \alpha, q} & \left(f ; \gamma_{n}, x\right)-f(x) \\
& =V_{n, \alpha, q}(f ; x)-f(x)-K_{n, \alpha, q}\left(f ; \gamma_{n}, x\right) .
\end{aligned}
$$

Using Corollary 10 and Lemma 5, we easily get the assertion (41).

Remark 12. Theorem 11 demonstrates that the generalized Baskakov operators $V_{n, \alpha, q}(f ; x)$ can be replaced by the truncated operators $B_{n, \alpha, q}\left(f ; \gamma_{n}, x\right)$ in a certain sense from the computational point of view. 


\section{Conflict of Interests}

The authors declare that there is no conflict of interests regarding the publication of this paper.

\section{Acknowledgments}

This work was supported by the National Natural Science Foundation of China (NSFC) under Grants nos. 41204041 and 61261043, by the EU FP7 Project EYE2E (269118), LIVCODE (295151), by the Science Research Project of Ningxia Higher Education Institutions of China under Grant no. NGY20140147, and in part by the Science Research Project of the State Ethnic Affairs Commission of China under Grant no. 14BFZ002.

\section{References}

[1] V. A. Baskakov, "An instance of a sequence of linear positive operators in the space of continuous functions," Doklady Akademii Nauk SSSR, vol. 113, pp. 249-251, 1957.

[2] M. Becker, "Global approximation theorems for SzaiszMirakjan and Baskakov operators in polynomial weight spaces," Indiana University Mathematics Journal, vol. 27, no. 1, pp. 127-142, 1978.

[3] Z. Ditzian, "On global inverse theorems of Szász and Baskakov operators," Canadian Journal of Mathematics, vol. 31, no. 2, pp. 255-263, 1979.

[4] N. K. Govil and V. Gupta, "Convergence rate for generalized Baskakov type operators," Nonlinear Analysis: Theory, Methods \& Applications, vol. 69, no. 11, pp. 3795-3801, 2008.

[5] V. Gupta, "An estimate on the convergence of Baskakov-Bezier operators," Journal of Mathematical Analysis and Applications, vol. 312, no. 1, pp. 280-288, 2005.

[6] V. Gupta and R. P. Agarwal, Convergence Estimates in Approximation Theory, Springer, New York, NY, USA, 2014.

[7] V. Gupta, P. N. Agrawal, and A. R. Gairola, "On the integrated Baskakov type operators," Applied Mathematics and Computation, vol. 213, no. 2, pp. 419-425, 2009.

[8] N. İspir, "On modified Baskakov operators on weighted spaces," Turkish Journal of Mathematics, vol. 25, no. 3, pp. 355-365, 2001.

[9] P. Sablonnière, "Approximation by Baskakov quasi-interpolants," http://arxiv.org/abs/1310.5093.

[10] Z. Walczak, "On the rate of convergence for modified Baskakov operators," Lithuanian Mathematical Journal, vol. 44, no. 1, pp. 102-107, 2004.

[11] Z. Walczak, "Baskakov type operators," The Rocky Mountain Journal of Mathematics, vol. 39, no. 3, pp. 981-993, 2009.

[12] Z. Walczak and V. Gupta, "A note on the convergence of Baskakov type operators," Applied Mathematics and Computation, vol. 202, no. 1, pp. 370-375, 2008.

[13] X.-M. Zeng and V. Gupta, "Rate of convergence of BaskakovBezier type operators for locally bounded functions," Computers \& Mathematics with Applications, vol. 44, no. 10-11, pp. 14451453, 2002.

[14] M. Gurdek, L. Rempulska, and M. Skorupka, "The Baskakov operators for functions of two variables," Collectanea Mathematica, vol. 50, no. 3, pp. 289-302, 1999.

[15] Y. Gao, "The approximation by a kind of generalized Baskakov operators of two variables on weighted space of polynomials,"
Journal of Henan Normal University (Natural Science), vol. 39, no. 5, pp. 6-9, 2011.

[16] Y. Gao, "The approximation properties by a kind of generalized Baskakov-Kantorovich operators," Journal of Inner Mongolia University (Natural Science), vol. 44, no. 3, pp. 234-238, 2013.

[17] J. Wang, H. Guo, and J. Jing, "Estimation of approximation with Jacobi weights by multivariate Baskakov operator," Journal of Function Spaces and Applications, vol. 2013, Article ID 939565, 6 pages, 2013.

[18] S. K. Serenbay, Ç. Atakut, and İ. Büyükyazıcı, “The generalized Baskakov type operators," Journal of Computational and Applied Mathematics, vol. 259, pp. 226-232, 2014.

[19] W. Z. Chen, Approximation Theory of Operators, Xiamen University Publishing House, Xiamen, China, 1989.

[20] X. Q. Hou and Y. C. Xue, "Approximation by generalized Baskakov operators," Chinese Quarterly Journal of Mathematics, vol. 5, no. 1, pp. 115-121, 1990.

[21] X. R. Zhang, "On uniform approximation characteristics with weights by generalized Baskakov operators," Journal of Ningxia University (Natural Science), vol. 17, no. 4, pp. 6-12, 1996.

[22] Y. Gao and Y. C. Xue, "On the approximation with weights for the generalized Baskakov operators," Journal of Ningxia University (Natural Science), vol. 25, no. 3, pp. 196-200, 2004.

[23] Z. Ditzian and V. Totik, Moduli of Smoothness, vol. 9 of Springer Series in Computational Mathematics, Springer, New York, NY, USA, 1987. 


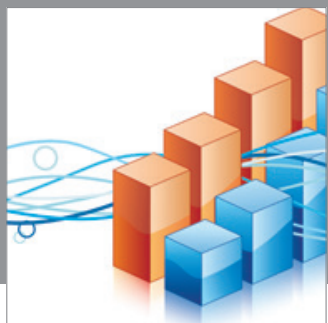

Advances in

Operations Research

mansans

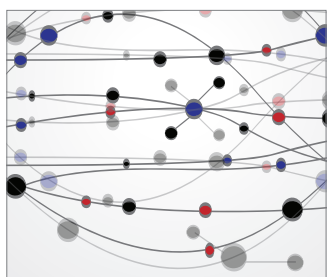

The Scientific World Journal
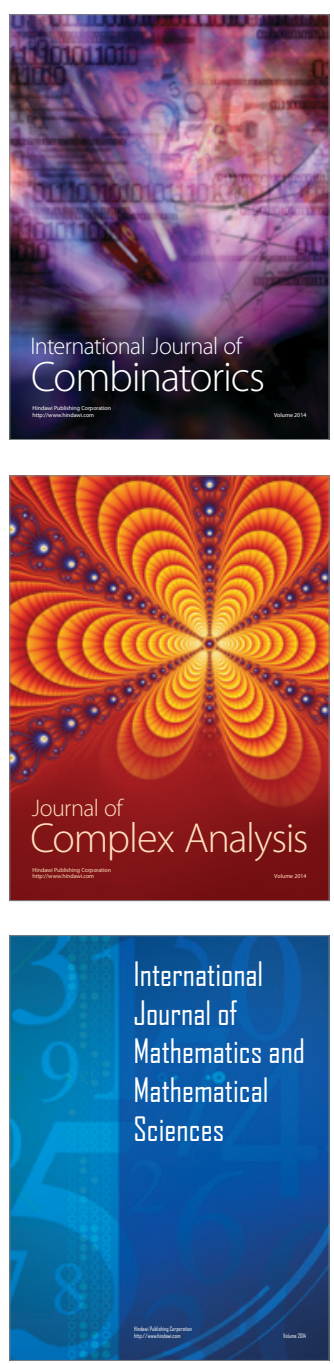
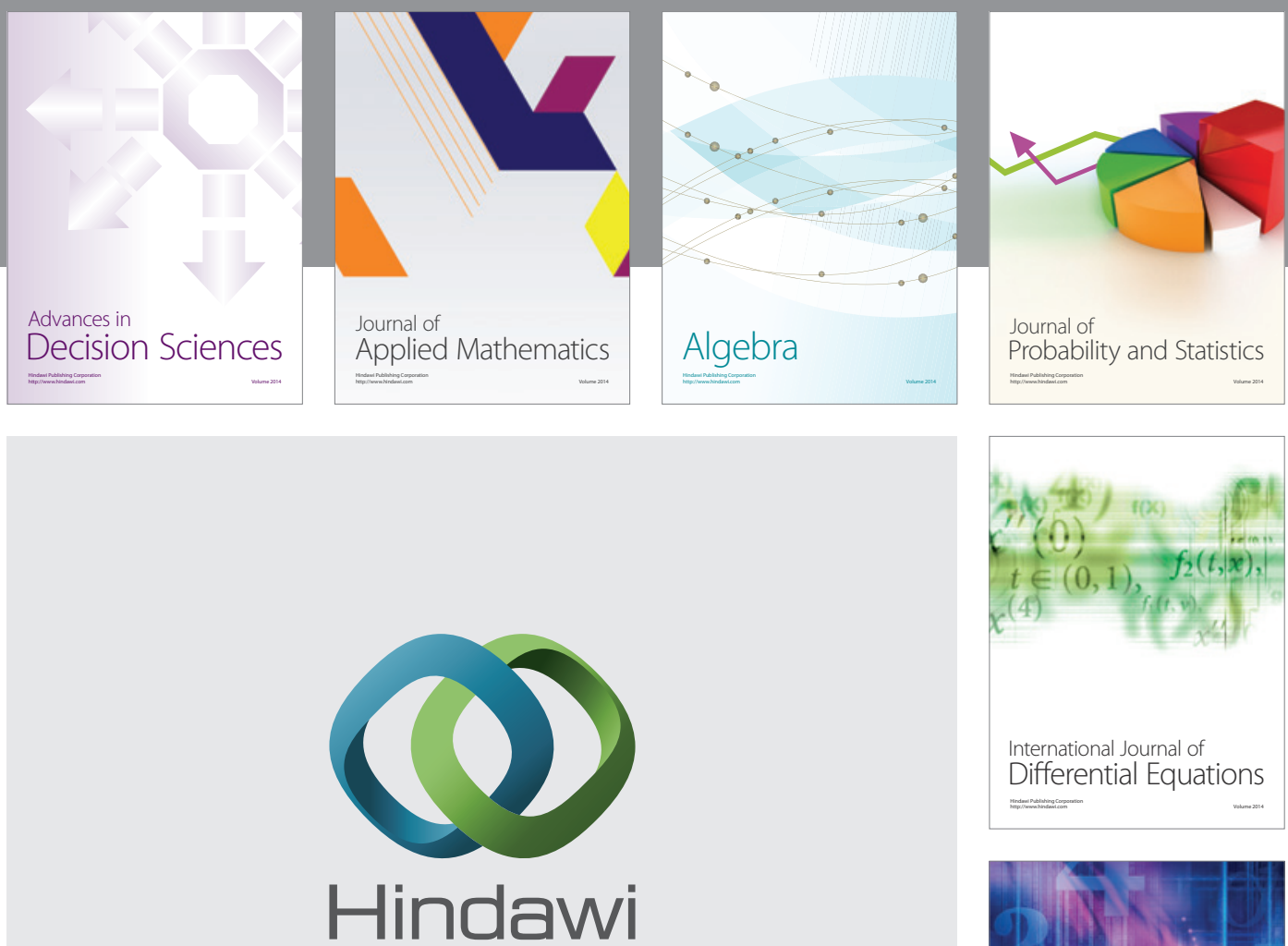

Submit your manuscripts at http://www.hindawi.com
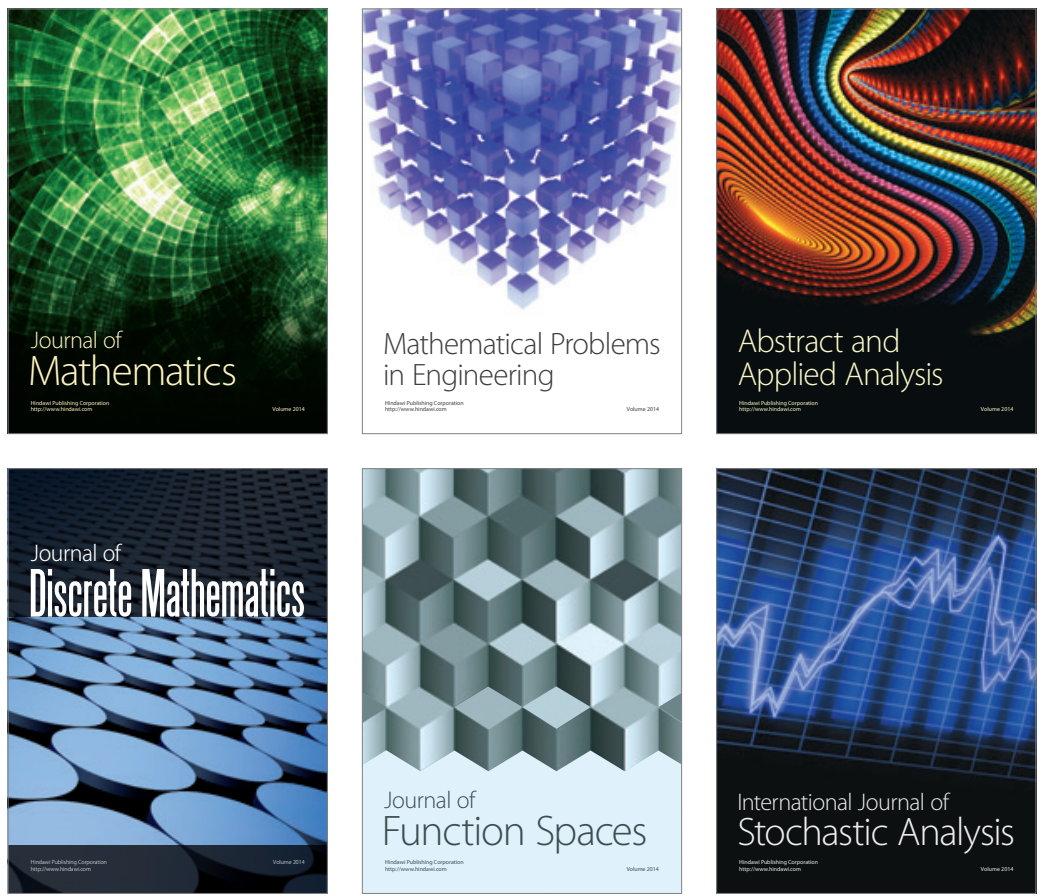

Journal of

Function Spaces

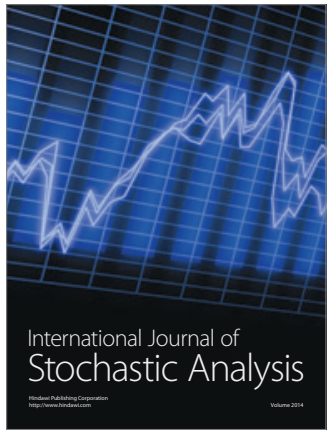

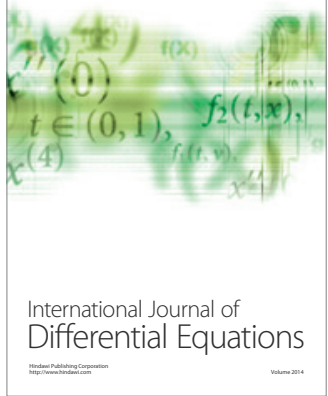
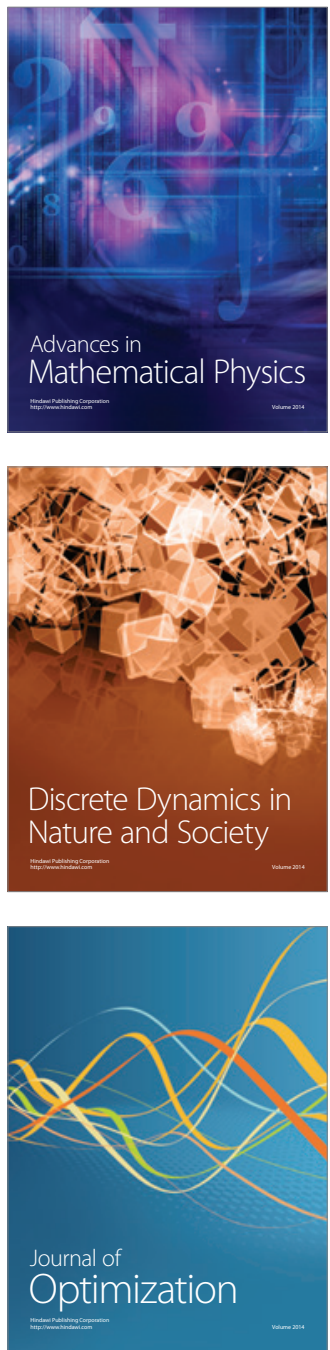